\title{
GENOME-WIDE IDENTIFICATION OF THE GATA TRANSCRIPTION FACTOR FAMILY IN Dichanthelium oligosanthes
}

\author{
Satyabrata Nanda ${ }^{1}$ (D), Gagan Kumar ${ }^{1}$, Sudheer Kumar Yadav², Sajid Hussain ${ }^{3}$
}

\author{
${ }^{1}$ MS Swaminathan School of Agriculture, Centurion University of Technology and Management, Odisha, India \\ ${ }^{2}$ Narayan Institute of Agriculture Sciences, Gopal Narayan Singh University, Bihar, India. \\ ${ }^{3}$ State Key Laboratory of Rice Biology, China National Rice Research Institute, Hangzhou, China. \\ Received - May 29, 2021; Revision - July 25, 2021; Accepted - August 16, 2021 \\ Available Online-August 30, 2021
}

DOI: http://dx.doi.org/10.18006/2021.9(4).407.416

\section{KEYWORDS \\ GATA \\ Transcription Factors \\ Genome-wide identification \\ $\mathrm{C}_{3}$ plants}

Dichanthelium oligosanthes

\begin{abstract}
The GATA transcription factors (TFs) play a crucial role in regulating various physiological processes in plants. Identification and characterization of the GATA TF family has been carried out in several important grass species, including rice, maize, and bamboo. However, no information is available on the GATA TFs in the $\mathrm{C}_{3}$ grass species Dichanthelium oligosanthes. In the current study, 31 GATA genes have been identified in the D. oligosanthes genome by stringent bioinformatics analysis. The exonintron arrangement analysis of the DoGATAs via the Gene Structure Display Server (GSDS 2.0) revealed the redundancy and differences in their gene structural organization. In addition, the sequence comparisons within the DoGATAs via BLAST revealed 11 numbers of putative paralogs. Similarly, the BLAST comparisons among the OsGATAs and DoGATAs resulted in the identification of 21 orthologs. Structural analysis of the identified DoGATAs through Simple Modular Architecture Research Tool (SMART), Conserved Domain Database (CDD), and Multiple Expectation Maximization for Motif Elicitation (MEME) revealed that all of them possess the signature GATA domain and the $\mathrm{C}-\mathrm{X}_{2}-\mathrm{C}-\mathrm{X}_{18}$ $\mathrm{C}-\mathrm{X}_{2}-\mathrm{C}$ consensus sequence. The phylogenetic analysis via MEGA divided the DoGATAs into four groups along with rice and Arabidopsis GATAs. In addition, the subcellular localization, gene ontology, and other peptide functional prediction results further supported the DoGATAs to be putative GATA genes. Moreover, the findings of this study can serve as a basic framework for the isolation and functional characterization of GATA genes in D. oligosanthes.
\end{abstract}

* Corresponding author

E-mail: satyabrata.nanda@cutm.ac.in; sbn.satyananda@gmail.com (Satyabrata Nanda)

Peer review under responsibility of Journal of Experimental Biology and Agricultural Sciences.

Production and Hosting by Horizon Publisher India [HPI] (http://www.horizonpublisherindia.in/).

All rights reserved.
All the articles published by Journal of Experimental Biology and Agricultural Sciences are licensed under a Creative Commons Attribution-NonCommercial 4.0 International License Based on a work at www.jebas.org.

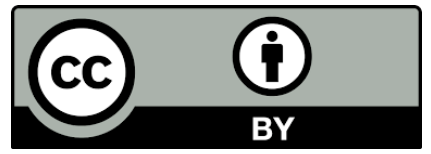




\section{Introduction}

Transcription factors (TFs) are the regulatory genetic elements that modulate the gene expressions during numerous physiological processes, including growth, development, and stress responses (Franco-Zorrilla et al., 2014). In plants, several TF families are with crucial roles, such as WRKY, bHLH (basic helix-loop-helix), MYB (V-myb avian myeloblastosis viral oncogene homolog), SPL (Squamosa-promoter binding protein-like), and GATA (GATAbinding factor). Amongst these, GATA is an important type of TF family having vital roles in hormonal signaling, development process, cellular metabolism, and stress response in plants (Liu et al., 2019a; An et al., 2020; Zhang et al., 2021). The GATA TFs are highly conserved across plant species, which has a typical DNA binding function onto the consensus A/T-GATA-A/G sequence (Lowry \& Atchley, 2000). Although initially these were discovered in fungi and animals, later they have identified in plants also. Daniel-Vedele \& Caboche (1993) identified the first plant GATA TF in tobacco plants. The subsequent identification and analysis of plant GATA factors suggested that they possess a tightly conserved consensus sequence of $\mathrm{C}-\mathrm{X}_{2}-\mathrm{C}-\mathrm{X}_{18}-\mathrm{C}-\mathrm{X}_{2}-\mathrm{C}$. Most plant GATA proteins have a single consensus domain, while some may contain more than two zinc finger domains or additional zinc finger loops (Reyes et al., 2004). Several GATA TFs have been identified in various plants, such as Arabidopsis, rice, wheat, poplar, tomato, soybean, and chili pepper (Reyes et al., 2004 Zhang et al., 2015; Yuan et al., 2018; Liu et al., 2019b; An et al., 2020; Yu et al., 2021). Additionally, their roles in plants, including germination, development, metabolism, signaling pathways, and stress response have also been investigated extensively (Liu et al., 2019a; Nutan et al., 2019; An et al., 2020). However, the identification and functional validation of the GATA TFs in many other plants are yet to be explored.

Dichanthelium oligosanthes is a perennial frost tolerant grass species found in North America. Unlike its other grass family relatives, including maize and sugarcane, $D$. oligosanthes use the C3 pathway for photosynthesis (Bräutigam et al., 2014). This lack of Kranz anatomy and the use of C3 pathway makes $D$. oligosanthes a promising candidate to be a model $\mathrm{C} 3$ plant that can be compared to the other $\mathrm{C} 4$ plants. The sequencing and availability of the draft genome of $D$. oligosanthes has opened new research avenues. Sequence information of plant genomes is crucial to perform the genome-wide gene identification and characterization, and have been successfully employed in numerous plants, including Arabidopsis, rice, wheat, maize, bamboo, cotton, tomato, chili pepper, and so on (Kumar et al., 2017; Wang et al., 2019; Li et al., 2020; Huang et al., 2020; Zheng et al., 2020; Liu et al., 2020a; Yang et al., 2021; Yu et al., 2021; Tiwari et al., 2021). In D. oligosanthes, the genome-wide identification of SPL and zinc finger proteins $\left(\mathrm{C}_{2} \mathrm{H}_{2}\right.$-ZFPs $)$ have already been reported (Hussain \& Nanda, 2019; Mahapatra et al., 2019). In this study, the genome-wide investigation of the GATA TFs in D. oligosanthes (named as DoGATAs) has been performed. Additionally, the structural organization of the identified GATA genes has been analyzed and their exon-intron arrangements are depicted. Further, different peptide properties of the identified putative DoGATAs have been also predicted. The evolutionary relationship within the putative DoGATAs and among other plant GATAs are analyzed by constructing a phylogenetic tree. In addition, the homologous GATAs, including both the paralogous DoGATA pairs and the orthologous with rice GATA are identified. Moreover, the functional attributes of the identified putative DoGATAs are predicted by the gene ontology analysis and subcellular localization predictions.

\section{Materials and Methods}

\subsection{Identification of the GATAs}

From the NCBI (http://www.ncbi.nlm.nih.gov/), the draft $D$. oligosanthes was obtained and the sequences were used in this study. The hidden Markov model (HMM) of the GATA domain (PF00320) was downloaded from the Pfam database (Mistry et al., 2021). Using the HMM file, candidate GATAs in D. oligosanthes were searched in the HMMER program with a threshold of Evalues $<10^{-5}$ (Wheeler \& Eddy, 2013). The retrieved sequences were screened by performing local BLASTP and the redundant sequences were removed. Further, all selected protein sequences were screened for the signature GATA domain by the Simple Modular Architecture Research Tool (SMART) and the Conserved Domain Database (CDD) (Lu et al., 2020; Letunic et al., 2021). Sequences, those not having the signature GATA domain were excluded from further downstream analysis. Subsequently, the different protein properties of the identified putative DoGATAs were predicted by using the ProtParam tool (Gasteiger et al., 2005).

\subsection{Sequence alignment and phylogenetic classification}

The identified putative DoGATA sequences were aligned amongst themselves to see the conserved regions in the sequences. In addition, GATA sequences from rice (OsGATAs) and Arabidopsis (AtGATAs) were collected from the Plant TF database and used for the alignment (Tian et al., 2019). The multiple sequence alignment (MSA) was performed on CLUSTAL Omega (Madeira et al., 2019). To deduce the evolutionary relationship among the DoGATAs and other plants GATAs, a phylogenetic tree was constructed by using the neighbor-joining method on MEGA v10 with 1000 bootstraps (Kumar et al., 2016). 


\subsection{Gene structure and motif analysis}

The identified DoGATA gene structures and the exon-intron organization were visualized by using the Gene Structure Display Server program (Hu et al., 2015). On the other hand, the de novo motif analysis of the putative DoGATAs was performed on the Multiple Expectation Maximization for Motif Elicitation (MEME) tool with the following parameters: motif repetition occurrences: any number, max motif numbers predicted: 5, and Minimum/Maximum motif width: 26/32 (Bailey et al., 2009).

\subsection{Paralogous and orthologous identification}

To find the paralogous DoGATAs, all identified DoGATA cDNA sequences were compared among themselves via a local BLAST alignment. The DoGATA sequence that exhibited $>40 \%$ similarity with $\geq 300$ bp sequence alignment was considered as a paralog (Blanc \& Wolfe, 2004). Conversely, each OsGATA cDNA sequence was searched against all DoGATA cDNAs by performing a local BLAST alignment to find out the orthologs. Alignments of $>300$ bp and with best hit scores were considered to be orthologs (Blanc \& Wolfe, 2004).

\subsection{Gene ontology and subcellular localization analysis}

The gene ontology (GO) analysis of the identified DoGATAs was performed by using their putative protein sequences. Briefly, each protein sequence was uploaded onto the DeepGO protein function prediction tool and the potential protein functions were predicted based on the DeepGO plus algorithm along with the GO classes (Kulmanov et al., 2018). In addition, the subcellular localization of DoGATAs was predicted by using the mGOASVM server dedicated to plants (Wan et al., 2012).

\section{Results}

The HMMER search with the GATA domain HMM file (PF00320) showed 34 hits with a threshold of e-values $<10^{-5}$. These sequences were collected and checked for the presence of the signature GATA domain with SMART and CDD tools. Out of 34 sequences, only 31 showed the presence of the required GATA domain. The sequences which contained the GATA domain were retained for further analysis and the remaining 3 sequences were discarded. These 31 sequences were then named as DoGATA1 to DoGATA31 for ease of downstream analysis. The peptide properties of all the 31 putative DoGATAs were predicted by using the Protparam tool. The DoGATAs showed a wide range of molecular weights (MW), the largest being DoGATA1 with a MW of $62.68 \mathrm{KDa}$ and the smallest being DoGATA31 with a MW of 16.26 KDa. Similarly, the CDS length and amino acid length of the identified DoGATAs has also exhibited a variable range with an average amino acid length of $\sim 317$ aa. Likewise, the isoelectric point (pI) range was found to be from 4.69 (DoGATA25) to 11.56 (DoGATA12) (Table 1). Moreover, all 31 DoGATAs exhibited a negative grand average of hydropathy (GRAVY) value indicating their hydrophilic nature.

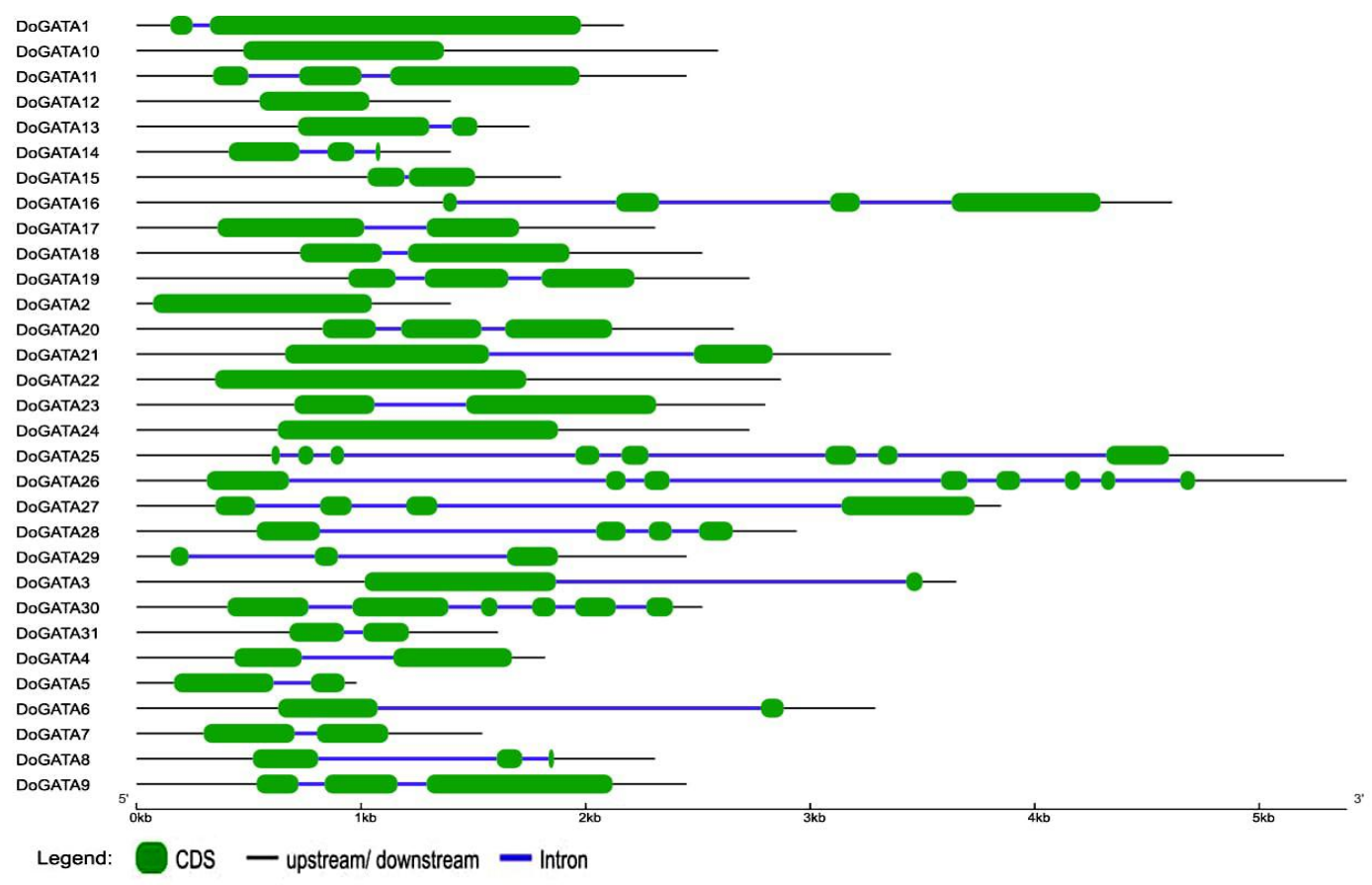

Figure 1 The structural (exon/intron) organization of DoGATA genes. The exons, introns, and UTR regions are represented by green boxes, blue lines, and black lines, respectively.

Journal of Experimental Biology and Agricultural Sciences http://www.jebas.org 
Table 1 The gene details and predicted protein properties of the 31 putative DoGATA genes in D. oligosanthes.

\begin{tabular}{|c|c|c|c|c|c|c|c|c|c|}
\hline Name & Gene Accessions & Exons & $\begin{array}{l}\text { CDS } \\
\text { in bp }\end{array}$ & $\begin{array}{l}\text { Size } \\
\text { (aa) }\end{array}$ & $\begin{array}{l}\text { MW } \\
(\mathrm{KDa})\end{array}$ & $\mathrm{pI}$ & $\begin{array}{l}\text { GRAVY } \\
\text { Score }\end{array}$ & Localization & ction \\
\hline DoGATA1 & LWDX02055130 & 2 & 1752 & 584 & 62.68 & 9.28 & -0.604 & Nucleus & GO:0009987 - cellular process, GO:0050896 - response to stimulus, GO:0008152 - metabolic process \\
\hline DoGATA2 & LWDX02028086 & 1 & 975 & 325 & 36.08 & 10.93 & -1.083 & Nucleus & GO:0009987 - cellular process, GO:0065007 - biological regulation, GO:0050896 - response to stimulus \\
\hline DoGATA3 & LWDX02026344 & 2 & 927 & 309 & 35.11 & 8.60 & -0.884 & Nucleus & GO:0009987 - cellular process, GO:0065007 - biological regulation, GO:0050789 - regulation of biol process \\
\hline DoGATA4 & LWDX02060644 & 2 & 828 & 276 & 28.53 & 8.25 & -0.240 & Nucleus & GO:0009987 - cellular process, GO:0065007 - biol regulation, GO:0050789 - regulation of biolprocess \\
\hline DoGATA5 & LWDX02007638 & 2 & 594 & 198 & 22.17 & 9.33 & -1.338 & Nucleus & GO:0065007 - biol regulation, GO:0050789 - regulation of biol process, GO:0009987 - cellular process \\
\hline DoGATA6 & LWDX02016181 & 2 & 678 & 226 & 23.37 & 8.68 & -0.300 & Nucleus & GO:0009987 - cellular process, GO:0032502 - develop process, GO:0050896 - response to stimulus \\
\hline DoGATA7 & LWDX02031362 & 2 & 723 & 241 & 24.69 & 7.50 & -0.307 & Nucleus & GO:0009987 - cellular process, GO:0050896 - response to stimulus, GO:0032502 - developmental process \\
\hline DoGATA8 & LWDX02024882 & 3 & 429 & 143 & 15.58 & 9.82 & -0.727 & Nucleus & GO:0065007 - biol regulation, GO:0050789 - regulation of biol process, GO:0009987 - cellular process \\
\hline DoGATA9 & LWDX02015205 & 3 & 1338 & 446 & 46.69 & 8.97 & -0.350 & Nucleus & GO:0009987 - cellular process, GO:0008152 - metabolic process, GO:0044237 - cellular metabolic process \\
\hline DoGATA10 & LWDX02056234 & 1 & 894 & 298 & 32.62 & 8.72 & -0.733 & Nucleus & $\begin{array}{c}\text { GO:0009987 - cellular process, GO:0006139 - nucleobase metabolic process, GO:0006725 - cellular aromatic } \\
\text { compound metabolic process }\end{array}$ \\
\hline DoGATA11 & LWDX02072364 & 3 & 1278 & 426 & 44.86 & 8.01 & -0.522 & cleus & GO:0009987 - cellular process, GO:0008152 - metabolic process, GO:0044237 - cellular metabolic process \\
\hline DoGATA12 & LWDX02032598 & 1 & 489 & 163 & 17.39 & 11.56 & -0.535 & Nucleus & GO:0009987 - cellular process, GO:0050896 - response to stimulus \\
\hline DoGATA13 & LWDX02018633 & 2 & 699 & 233 & 24.24 & 7.10 & -0.418 & Nucleus & GO:0009987 - cellular process, GO:0008152 - metabolic process, GO:0071704 - organic metabolic process \\
\hline DoGATA14 & LWDX02065998 & 3 & 456 & 152 & 16.78 & 9.78 & -0.967 & Nucleus & GO:0009987 - cellular process, GO:0065007 - biological regulation, GO:0050789 - regulation of biol process \\
\hline DoGATA16 & LWDX02005501 & 4 & 1071 & 357 & 38.15 & 4.93 & -0.661 & cleus & GO:0009987 - cellular process, GO:0008152 - metabolic process, GO:0044237 - cellular metabolic process \\
\hline DoGATA17 & LWDX02043815 & 2 & 1068 & 356 & 38.11 & 8.77 & -0.585 & cleus & GO:0009987 - cellular process, GO:0050896 - response to stimulus, GO:0065007 - biological regulation \\
\hline DoGATA18 & LWDX02058546 & 2 & 1080 & 360 & 37.07 & 7.34 & -0.484 & Nucleus & GO:0009987 - cellular process, GO:0050896 - response to stimulus, GO:0008152 - metabolic process \\
\hline DoGATA19 & LWDX02043834 & 3 & 999 & 333 & 36.11 & 9.45 & -0.425 & Nucleus & GO:0009987 - cellular process, GO:0065007 - biological regulation, GO:0050789 - regulation of biol process \\
\hline DoGATA20 & LWDX02025796 & 3 & 1074 & 358 & 38.21 & 9.10 & -0.379 & Nucleus & GO:0009987 - cellular process, GO:0032502 - develop process, GO:0065007 - biological regulation \\
\hline DoGATA21 & LWDX02013367 & 2 & 1260 & 420 & 43.91 & 5.27 & -0.341 & Nucleus & GO:0009987 - cellular process, GO:0008152 - metabolic process, GO:0044237 - cellular metabolic process \\
\hline DoGATA22 & LWDX02045989 & 1 & 1389 & 463 & 49.36 & 6.87 & -0.471 & Nucleus & GO:0009987 - cellular process, GO:0008152 - metabolic process, GO:0044237 - cellular metabolic process \\
\hline DoGATA23 & LWDX02024355 & 2 & 1203 & 401 & 41.21 & 5.75 & -0.376 & Nucleus & GO:0009987 - cellular process, GO:0050896 - response to stimulus, GO:0032502 - developmental process \\
\hline DoGATA24 & LWDX02030349 & 1 & 1251 & 417 & 44.55 & 7.55 & -0.491 & Nucleus & GO:0009987 - cellular process, GO:0032502 - develop process, GO:0065007 - biological regulation \\
\hline DoGATA25 & LWDX02025210 & 8 & 894 & 298 & 31.62 & 4.69 & -0.658 & Nucleus & GO:0009987 - cellular process, GO:0065007 - biological regulation, GO:0050789 - regulation of biol process \\
\hline DoGATA26 & LWDX02036020 & 8 & 987 & 329 & 35.32 & 8.66 & -0.507 & Nucleus & GO:0009987 - cellular process, GO:0065007 - biological regulation, GO:0008152 - metabolic process \\
\hline DoGATA27 & LWDX02046859 & 4 & 1071 & 357 & 39.47 & 7.10 & -0.285 & Nucleus & GO:0009987 - cellular process, GO:0032502 - develop process, GO:0065007 - biological regulation \\
\hline DoGATA28 & LWDX02019626 & 4 & 666 & 222 & 24.09 & 7.51 & -0.725 & Nucleus & GO:0009987 - cellular process, GO:0008152 - metabolic process, GO:0065007 - biological regulation \\
\hline DoGATA29 & LWDX02052907 & 3 & 594 & 198 & 21.84 & 5.61 & -0.785 & Nucleus & GO:0009987 - cellular process, GO:0008152 - metabolic process, GO:0044237 - cellular metabolic process \\
\hline DoGATA30 & LWDX02037984 & 6 & 1266 & 422 & 46.84 & 6.90 & -0.791 & Nucleus & GO:0009987 - cellular process, GO:0006139 - metabolic process, GO:0006725 - cellular metabolic process \\
\hline
\end{tabular}

Journal of Experimental Biology and Agricultural Sciences

http://www.jebas.org 
The genetic structure and exon-intron organizations provide a better understanding of the genes. The cDNA sequences and open reading frames (ORFs) were identified for all 31 DoGATAs. The gene structures of the identified DoGATAs were analyzed by the GSDS 2.0 tool. The results revealed the number of exons and introns for all 31 DoGATA genes. The number exons in these genes ranged from 1 (DoGATA2, DoGATA10, DoGATA12, DoGATA22, and DoGATA24) to 8 (DoGATA25 and DoGATA26).Out of the 31 identified DoGATAs, 13 had 2 exons, whereas 4 had no introns at all (Figure 1). On the other hand, the MSA and de novo motif analysis of the DoGATAs revealed that all of them possessed the signature GATA motif with a conserved consensus of C- $\mathrm{X}_{2}-\mathrm{C}-\mathrm{X}_{18}-$ $\mathrm{C}-\mathrm{X}_{2}-\mathrm{C}$ (Figure 2).

The evolutionary relationship of the DoGATAs within and among other plant GATAs was analyzed by constructing a phylogenetic

A)

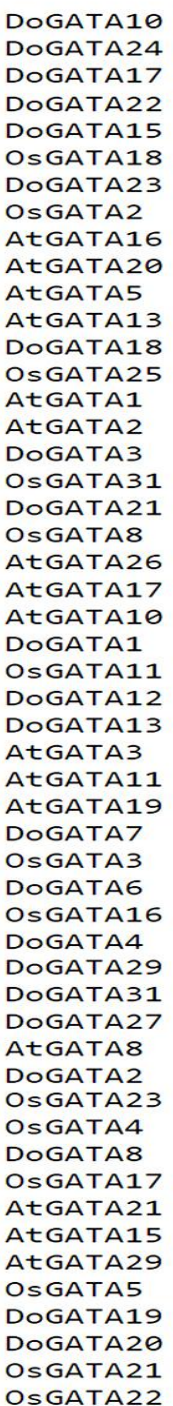

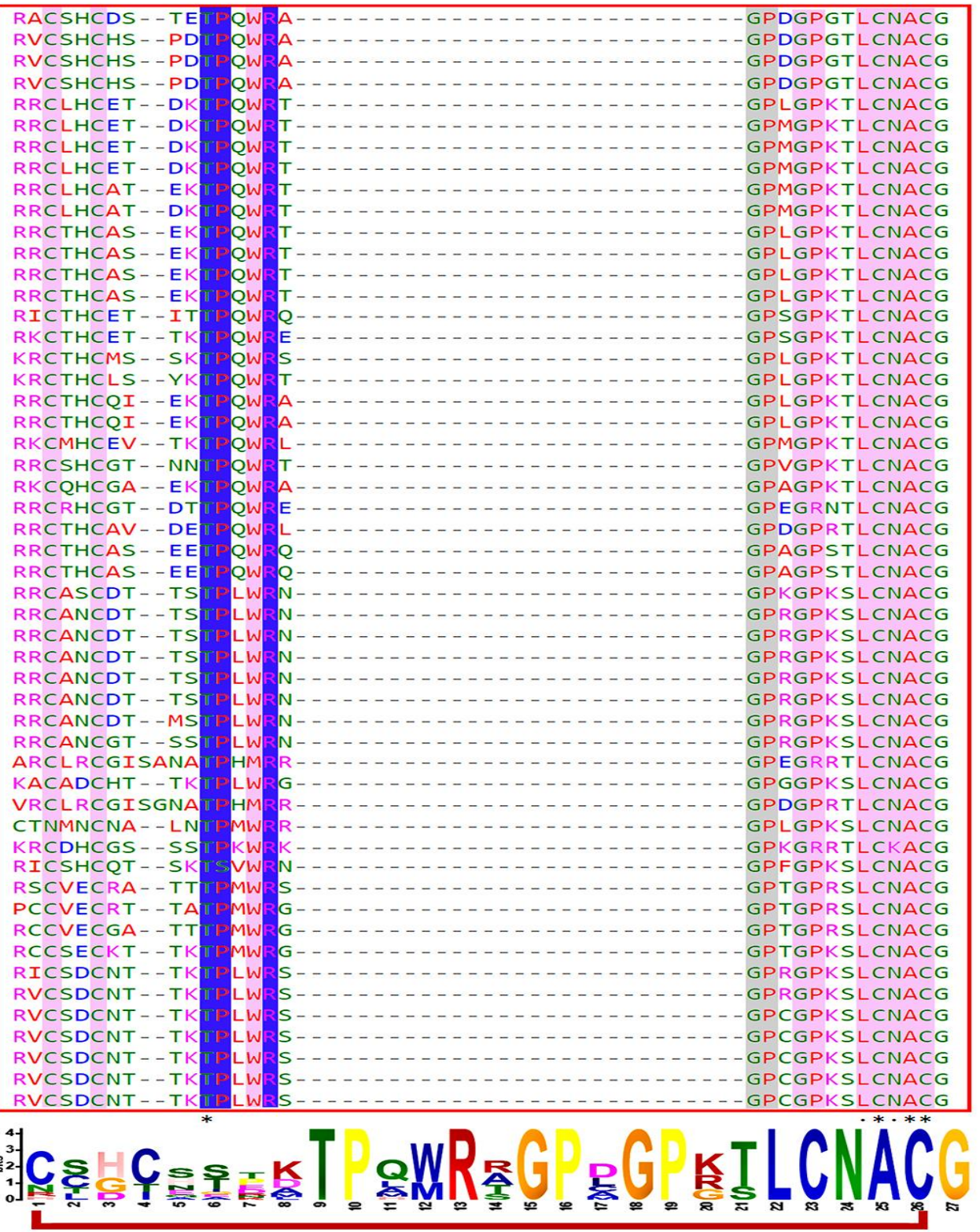

GATA (C-X2-C-X18-C-X2-C) motif

Figure 2 A) Multiple sequence alignment of the GATA sequences of rice, Arabidopsis, and D. oligosanthes. The amino acid positions and signature GATA motif are marked with the red color box and important positional motifs are highlighted in different colors. B) The GATA motif sequence logo as obtained from the structural analysis of the DoGATAs by the MEME suite. 
tree by employing the neighbor-joining algorithm on MEGA v10. In total, 31 DoGATA, 31 OsGATA, and 41 AtGATA protein sequences were used to align and construct the phylogenetic tree (Figure 3). All the sequences were clustered into four distinct groups (A-D). Group A has the highest number of GATA sequences, followed by $\mathrm{B}, \mathrm{C}$, and $\mathrm{D}$. Only one of the $D$. oligosanthes GATA (DoGATA2) was present in group D. Further, the screening for identification of paralogous pairs in the identified DoGATAs revealed that there are 11 paralogous genes among the 31 DoGATAs (Table 2). Similarly, a comparison of the DoGATAs with the OsGATAs resulted in the identification of 21 pairs of orthologous genes. Additionally, the functional annotations of the DoGATAs via the DeepGo analysis were performed. The results of the study suggested that the DoGATAs might majorly be involved in the cellular processes, such as developmental processes, metabolic processes, and response to stimuli (Table 1). Further, the GO term "GO:0005488" was associated with all DoGATAs. Moreover, the prediction of the subcellular localization of all 31 DoGATA revealed that they all might be localized in the nucleus.

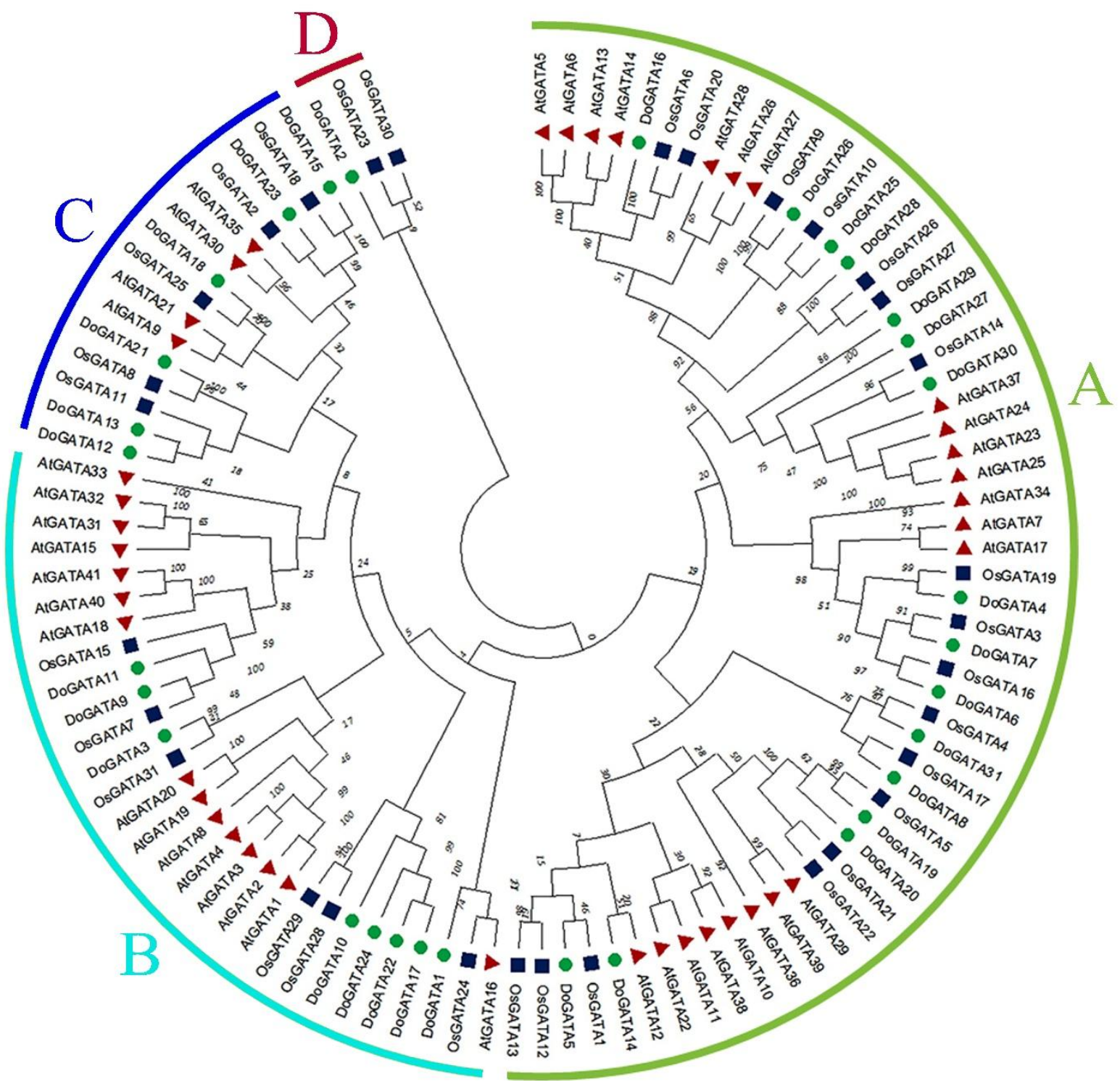

Figure 3 Phylogenetic analysis of the GATA TFs in D. ologosanthes, rice, and Arabidopsis. The tree has been constructed by using the protein sequences of the aforementioned plant GATAs by employing the neighbor-joining method on MEGA v10 with 1000 bootstrap replicates. Four major phylogenetic groups are indicated by letters A to D. The green solid circles denote the DoGATAs, blue squares denote the OsGATAs, and the maroon triangles denote the AtGATAs, respectively.

Journal of Experimental Biology and Agricultural Sciences http://www.jebas.org 
Table 2 A list of the paralogous (Do-Do) and orthologous (Do-Os) GATA gene pairs in D. oligosanthes and rice.

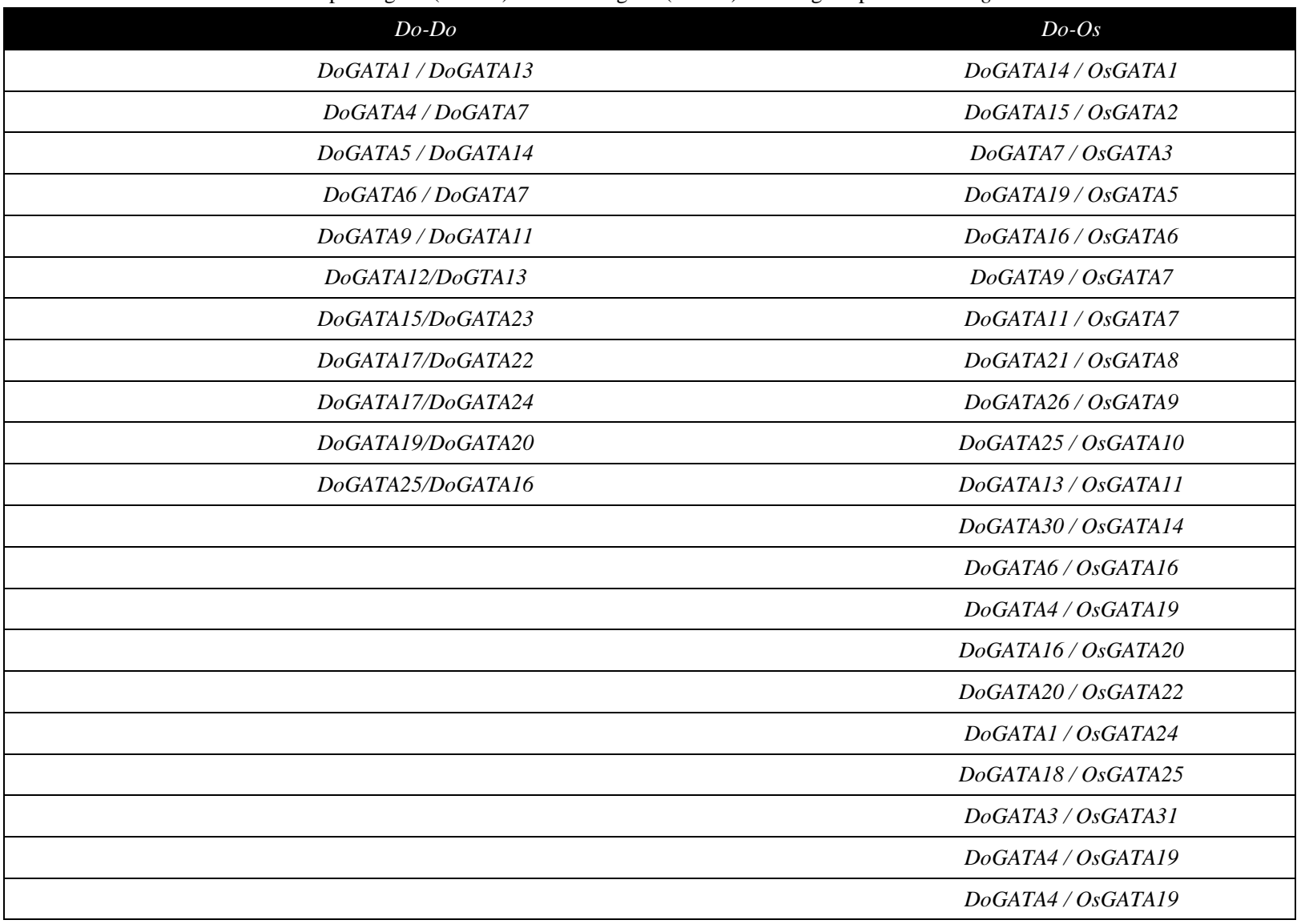

\section{Discussion}

The Heller's rosette grass (D. oligosanthes) is a $\mathrm{C}_{3}$ panicoid grass species that serves as an important candidate to study the $\mathrm{C}_{3}$ photosynthetic pathway and compare it with the $\mathrm{C}_{4}$ grasses. Most of these comparative studies were focused on the crop species, including rice $\left(\mathrm{C}_{3}\right)$ and maize, sugarcane, and sorghum $\left(\mathrm{C}_{4}\right)$ (Aliscioni et al., 2012). Thus, new experimental researches covering both in silico predictions and wet lab validations will be immensely helpful in providing vital insights into this important grass species. As the reports on the draft genome and the comparative RNA sequencing data of $D$. oligosanthes are available in the public domain, the present scenario is conducive enough to perform the genome-wide identification of gene or TF families (Hussain \& Nanda, 2019; Mahapatra et al., 2019). In this study, the stringent and methodological in silico analysis has resulted in the identification of 31 GATA TFs in D. oligosanthes (DoGATA1-31). The total number of GATA TFs identified in D. oligosanthes is the same as its C3 relative rice and shares sequence similarities. Additionally, the gene structure organization revealed that the genetic organization and intron-exon numbers greatly vary in the DoGATAs. This variation in the gene structure and exon numbers among GATAs has been reported in several plants, such as soybean, rapeseeds, bamboo, and pepper (Zhang et al., 2015; Wang et al., 2019; Zhu et al., 2020; Yu et al., 2021). These differences in gene and protein structures among the DoGATAs could result in functional divergences.

The structural analysis of the DoGATAs revealed that all 31 identified GATAs contain the conserved consensus of C- $\mathrm{X}_{2}-\mathrm{C}-\mathrm{X}_{18}$ $\mathrm{C}-\mathrm{X}_{2}$-C. On the phylogenetic tree, 31 DoGATA among rice and Arabidopsis GATAs were divided into 4 distinct groups (A-D). Previous reports suggest that the classification of GATA factors into 4 groups is mostly seen in the dicots, such as Arabidopsis, tomato, and cotton (Reyes et al., 2004; Yuan et al., 2018; Zhang et al., 2020). In contrast, monocot GATAs are divided into 3 groups as in rice (Reyes et al., 2004). In our study, the classification of the DoGATAs into 4 groups differs from these reports, however, similar to the results obtained by Wang et al. (2019) in bamboo plants. On the other hand, apart from the difference in classification, the DoGATAs exhibited greater evolutionary conservation patterns as compared to the $\mathrm{A}, \mathrm{B}$, and $\mathrm{C}$ group GATAs in Arabidopsis and rice. Thus, it could be inferred that the majority of the DoGATAs are conserved as compared to other 
grass species in the due course of evolution. Several paralogous pairs of genes arise due to the duplication of gene events in the due course of evolution (Lallemand et al., 2020). Additionally, these events play key roles in the species adaptations and developments in the environment (Lallemand et al., 2020). To get additional insights into the evolution of the DoGATAs, we have analyzed the paralogous and orthologous pairs in DoGATAs and in between OsGATAs and DoGATAs, respectively. The obtained results suggested that the GATA gene family in D. oligosanthes has experienced a large-scale gene duplication event, as we found 11 out of 31 GATAs to be putative paralogs. On the other hand, the identification of 21 orthologous pairs with OsGATAs indicated the tight conservancy nature of the GATA TFs in the grass species.

To get additional insights on the putative functions of DoGATAs, the peptide properties, subcellular localization, and GO predictions were performed. All of the DoGATAs had a negative GRAVY value indicating possibly their hydrophilic nature. Additionally, all of them were predicted to get localized inside the nucleus (Table 1). Being transcription factors, the GATAs mostly localize in the nucleus, where they regulate the downstream gene expressions by binding with their DNA binding domain (Zhang et al., 2020; Nutan et al., 2019). Further, all the 31 DoGATAs were predicted to have a DNA binding activity and associated with the GO term "GO:0005488". These results collectively suggested that the localization and putative function of the DoGATAs. Moreover, GO predictions indicated that several of the identified DoGATAs might be involved in key physiological processes, including metabolic pathways, cellular processing, and response to stimuli (Liu et al., 2019a; Liu et al., 2020b; Zhu et al., 2020; Zhang et al., 2020; Zhang et al., 2021). Functional validation and in-depth analysis of the identified GATAs in D. oligosanthes will confirm their roles in the plant.

\section{Conclusion}

The present study reported that the genome-wide identification and analysis of the GATA TF family in $D$. oligosanthes, an important $\mathrm{C}_{3}$ grass. In addition, the systematic and stringent in silico analysis resulted in the identification of 31 GATAs in D. oligosanthes. Their putative protein properties predictions revealed the diverse structural ranges and suggested their possible hydrophilic nature. The exon-intron arrangements revealed the structural variations in the gene structures of DoGATAs. Furthermore, the phylogenetic analysis clustered the DoGATAs among OsGATA and AtGATAs into four distinct groups. The analysis of paralogous pairs among the DoGATAs hinted towards the possible early and large-scale gene duplication event in the GATA TF family in D. oligosanthes. On the other hand, the identification of 21 pairs of orthologs with rice GATAs indicated the conserved nature of the GATAs in the grass family. Furthermore, the MSA and the de novo motif analysis revealed that all 31 DoGATAs possess the signature GATA domain and consensus sequence. The GO analysis along with the sub-cellular localization prediction and other protein properties suggested the putative roles of the DoGATAs. Moreover, this study can serve as a basic framework for future functional validation and in-depth analysis of the GATA genes in $D$. oligosanthes.

\section{Acknowledgments}

SN and GK thank Centurion University of Technology and Management for the necessary facilities and support. SKY is thankful to Gopal Narayan Singh University for its encouragement and support. SH is thankful for the award of Postdoctoral Fellowship from the Chinese Academy of Agricultural Sciences.

\section{Conflict of interest}

The authors declare no conflicts of interest.

\section{References}

Aliscioni S, Bell HL, Besnard G, et al. (2012). New grass phylogeny resolves deep evolutionary relationships and discovers C4 origins. New Phytologist 193(2), 304- 312. https://doi.org/10.1111/ j.1469-8137.2011.03972.x

An Y, Zhou Y, Han X, et al. (2020) The GATA transcription factor GNC plays an important role in photosynthesis and growth in poplar. Journal of Experimental Botany 71:1969-1984.

Bailey TL, Boden M, Buske FA, et al. (2009) MEME SUITE: tools for motif discovery and searching. Nucleic Acids Research 37:W202-W208.

Blanc G, Wolfe KH (2004) Widespread paleopolyploidy in model plant species inferred from age distributions of duplicate genes. Plant Cell 16(7):1667-1678.

Bräutigam A, Schliesky S, Külahoglu C, et al. (2014) Towards an integrative model of $\mathrm{C} 4$ photosynthetic subtypes: insights from comparative transcriptome analysis of NAD-ME, NADP-ME, and PEP-CK C4 species. Journal of Experimental Botany 65:35793593.

Daniel-Vedele F, Caboche M (1993) A tobacco cDNA clone encoding a GATA-1 zinc finger protein homologous to regulators of nitrogen metabolism in fungi. Molecular Genetics and Genomics 240:365-373.

Franco-Zorrilla JM, López-Vidriero I, Carrasco JL, et al. (2014) DNA-binding specificities of plant transcription factors and their potential to define target genes. Proceedings of the National Academy of Sciences of the United States of America 111:23672372. 
Gasteiger E, Hoogland C, Gattiker A, et al. (2005) Protein Identification and Analysis Tools on the ExPASy Server. In: The Proteomics Protocols Handbook. Humana Press, Totowa, NJ, Pp 571-607.

Hu B, Jin J, Guo AY, et al. (2015) GSDS 2.0: an upgraded gene feature visualization server. Bioinformatics 31:1296-1297.

Huang J, Sun W, Ren J, et al. (2020) Genome-Wide Identification and Characterization of Actin-Depolymerizing Factor (ADF) Family Genes and Expression Analysis of Responses to Various Stresses in Zea Mays L. International Journal of Molecular Sciences 21:1751.

Hussain S, Nanda S (2019) Genome-wide identification of the SPL gene family in Dichanthelium oligosanthes. Bioinformation $15: 165-171$

Kulmanov M, Khan MA, Hoehndorf R (2018) DeepGO: predicting protein functions from sequence and interactions using a deep ontology-aware classifier. Bioinformatics 34:660-668.

Kumar M, Gho YS, Jung KH, Kim SR (2017) Genome-Wide Identification and Analysis of Genes, Conserved between japonica and indica Rice Cultivars, that Respond to Low-Temperature Stress at the Vegetative Growth Stage. Frontiers in Plant Science 8. https://doi.org/10.3389/fpls.2017.01120.

Kumar S, Stecher G, Tamura K (2016) MEGA7: Molecular Evolutionary Genetics Analysis Version 7.0 for Bigger Datasets. Molecular Biology and Evolution 33:1870-1874.

Lallemand T, Leduc M, Landès C, Rizzon C, Lerat E (2020) An Overview of Duplicated Gene Detection Methods: Why the Duplication Mechanism Has to Be Accounted for in Their Choice. Genes 11(9):1046.

Letunic I, Khedkar S, Bork P (2021) SMART: recent updates, new developments and status in 2020. Nucleic Acids Research 49:D458-D460.

Li Y, Song Q, Zhang Y, et al. (2020) Genome-wide identification, characterization, and expression patterns analysis of the SBP-box gene family in wheat (Triticum aestivum L.). Scientific Reports 10:17250.

Liu C, Li Z, Dou L, et al. (2020a) A genome-wide identification of the BLH gene family reveals BLH1 involved in cotton fiber development. Journal of Cotton Research 3:26.

Liu H, Li T, Wang Y, et al. (2019a) TaZIM-A1 negatively regulates flowering time in common wheat (Triticum aestivum L.). Journal of Integrative Plant Biology 61:359-376.
Liu X, Zhu X, Wei X, et al. (2020b) The wheat LLM-domaincontaining transcription factor TaGATA1 positively modulates host immune response to Rhizoctonia cerealis. Journal of Experimental Botany 71(1):344-355.

Liu Y, Patra B, Pattanaik S, et al. (2019b) GATA and Phytochrome Interacting Factor Transcription Factors Regulate Light-Induced Vindoline Biosynthesis in Catharanthus roseus. Plant Physiology 180:1336-1350.

Lowry JA, Atchley WR (2000) Molecular Evolution of the GATA Family of Transcription Factors: Conservation Within the DNABinding Domain. Journal of Molecular Evolution 50:103-115.

Lu S, Wang J, Chitsaz F, et al. (2020) CDD/SPARCLE: the conserved domain database in 2020. Nucleic Acids Research 48:D265-D268.

Madeira F, Park Y mi, Lee J, et al. (2019) The EMBL-EBI search and sequence analysis tools APIs in 2019. Nucleic Acids Research 47:W636-W641.

Mahapatra M, Mahanty B, Joshi RK (2019) Genome wide identification and functional assignments of $\mathrm{C} 2 \mathrm{H} 2$ Zinc-finger family transcription factors in Dichanthelium oligosanthes. Bioinformation 15:689-696.

Mistry J, Chuguransky S, Williams L, et al. (2021) Pfam: The protein families database in 2021. Nucleic Acids Research 49:D412-D419.

Nutan KK, Singla-Pareek SL, Pareek A (2019) The Saltol QTLlocalized transcription factor OsGATA8 plays an important role in stress tolerance and seed development in Arabidopsis and rice. Journal of Experimental Botany 71(2):684-698.

Reyes JC, Muro-Pastor MI, Florencio FJ (2004) The GATA Family of Transcription Factors in Arabidopsis and Rice. Plant Physiology 134:1718-1732.

Tian F, Yang D-C, Meng Y-Q, et al. (2019) PlantRegMap: charting functional regulatory maps in plants. Nucleic Acids Research 48(1):D1104-D1113.

Tiwari S, Muthamilarasan M, Lata C (2021) Genome-wide identification and expression analysis of Arabidopsis GRAMdomain containing gene family in response to abiotic stresses and PGPR treatment. Journal of Biotechnology 325:7-14.

Wan S, Mak M-W, Kung SY (2012) mGOASVM: Multi-label protein subcellular localization based on gene ontology and support vector machines. BMC Bioinformatics 13:290. 
Wang T, Yang Y, Lou S, et al. (2019) Genome-Wide Characterization and Gene Expression Analyses of GATA Transcription Factors in Moso Bamboo (Phyllostachys edulis). International Journal of Molecular Sciences 21:14.

Wheeler TJ, Eddy SR (2013) nhmmer: DNA homology search with profile HMMs. Bioinformatics 29:2487-2489.

Yang H, Sun Y, Wang H, et al. (2021) Genome-wide identification and functional analysis of the ERF2 gene family in response to disease resistance against Stemphylium lycopersici in tomato. BMC Plant Biology 21:72.

Yu C, Li N, Yin Y, et al. (2021) Genome-wide identification and function characterization of GATA transcription factors during development and in response to abiotic stresses and hormone treatments in pepper. Journal of Applied Genetics 62:265-280.

Yuan Q, Zhang C, Zhao T, et al. (2018) A genome-wide analysis of GATA transcription factor family in tomato and analysis of expression patterns. International Journal of Agriculture and Biology 20:1274-1282.
Zhang C, Hou Y, Hao Q, et al. (2015) Genome-Wide Survey of the Soybean GATA Transcription Factor Gene Family and Expression Analysis under Low Nitrogen Stress. PLoS One 10:e0125174.

Zhang C, Huang Y, Xiao Z, et al. (2020) A GATA Transcription Factor from Soybean (Glycine max) Regulates Chlorophyll Biosynthesis and Suppresses Growth in the Transgenic Arabidopsis thaliana. Plants 9:1036.

Zhang $\mathrm{H}$, Wu T, Li Z, et al. (2021) OsGATA16, a GATA Transcription Factor, Confers Cold Tolerance by Repressing OsWRKY45-1 at the Seedling Stage in Rice. Rice 14:42.

Zheng W, Zhang Y, Zhang Q, et al. (2020) Genome-Wide Identification and Characterization of Hexokinase Genes in Moso Bamboo (Phyllostachys edulis). Frontiers in Plant Science 11. https://doi.org/10.3389/fpls.2020.00600.

Zhu W, Guo Y, Chen Y, et al. (2020) Genome-wide identification, phylogenetic and expression pattern analysis of GATA family genes in Brassica napus. BMC Plant Biology 20:543. 